\title{
SUPERVIVENCIA DE IMPLANTES DENTALES Y COMPLICACIONES INTRA Y POSOPERATORIAS EN ELEVACIÓN DE SENO MAXILAR CON VENTANA LATERAL E IMPLANTE DENTAL SIMULTÁNEO EN REBORDES CON REABSORCIÓN SEVERA
}

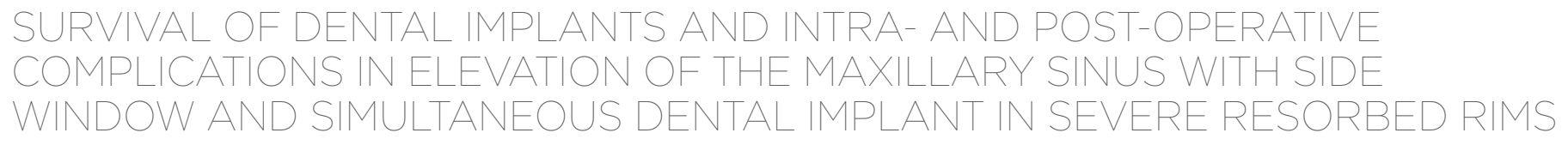

\section{Fabio Andrés Jiménez-Castellanos ${ }^{1,3}$ * (i)}

fjimenezc@unicoc.edu.co

Paola Castro-Pereira2 ${ }^{2}$ paola.cp01@gmail.com

Asterlis Buitrago-Osuna ${ }^{3}$ (1) asterhom@hotmail.com

Christian Andrés Huertas-Torres 3 (1) andre671@outlook.es

Artículo recibido: 30/04/2020

Arbitrado por pares

Artículo aceptado: 16/07/2020

Artículo publicado: 24/08/2020

\section{* Autor corresponsal:}

Fabio Andrés Jiménez-Castellanos

fjimenezc@unicoc.edu.co

Citar como: Jiménez-Castellanos FA, Castro-Pereira P, Buitrago-Osuna A, Huertas-Torres CA. Supervivencia de implantes dentales y complicaciones intra y posoperatorias en elevación de seno maxilar con ventana lateral e implante dental simultáneo en rebordes con reabsorción severa. Una revisión de la literatura. Rev Cient Odontol (Lima). 2020; 8 (2): e025.

DOI: 10.21142/2523-2754-0802-2020-025

\section{RESUMEN}

Objetivo: El posicionamiento de implantes dentales simultáneo a la elevación del seno maxilar en rebordes con reabsorción ósea severa $(\leq 4 \mathrm{~mm})$ es una técnica quirúrgica que disminuye los tiempos operatorios. Sin embargo, es considerada sensible por ser dependiente del operador y, en el caso que no se dé un manejo adecuado, puede presentar complicaciones. En este estudio se realiza una revisión de la literatura sobre la supervivencia de los implantes dentales y las complicaciones intra y posoperatorias en procedimientos de elevación del seno maxilar con la colocación simultánea de implante dental en rebordes con reabsorción ósea severa. Materiales y métodos: Se realizó una búsqueda bibliográfica de la literatura publicada en los últimos 10 años, durante el periodo de mayo y junio del 2019, en las bases de datos Medline-PubMed, EBSCOhost y Scopus. Esta se complementó con una búsqueda manual en revistas especializadas en periodoncia y cirugía oral Q1, posicionadas en el top 5 del 2018 en www.scimagojr.com. Se incluyeron ensayos clínicos y estudios prospectivos y retrospectivos. Se identificó 2562 artículos científicos. Tras el análisis de los títulos, la lectura de los resúmenes y los textos completos, se seleccionaron 6 artículos para el análisis de la técnica quirúrgica y 35 para complementar la información. Conclusiones: La colocación simultánea de implantes dentales a la elevación del seno maxilar es una de las técnicas utilizadas para restituir la función en el maxilar posterior. La complicación intraoperatoria más frecuente es la perforación de la membrana sinusal y la posoperatoria es la infección asociada con perforación de la membrana, o la migración del implante al seno maxilar. La supervivencia promedio del implante observada en los estudios es mayor al $94 \%$.

Palabras clave: seno maxilar, implantes dentales, complicaciones intraoperatorias, complicaciones posoperatorias

\begin{abstract}
Aim: The positioning of dental implants simultaneous to the elevation of the maxillary sinus in ridges with severe bone resorption $(\leq 4 \mathrm{~mm})$ is a surgical technique that reduces operative times. However, it is considered sensitive, being dependent on the operator, and in the absence of appropriate handling, complications can occur. This study aimed to provide a review of the literature on the survival of dental implants and intra- and post-operative complications in maxillary sinus lift procedures with the simultaneous placement of a dental implant in ridges with severe bone resorption. Materials and methods: A bibliographic search of the literature published in the last 10 years in the Medline-Pubmed, EBSCO HOST and SCOPUS databases was carried out from May to June 2019. This was complemented with a manual search in journals specialized in Periodontology and Oral Surgery Q1, positioned in the Top 5 of the year 2018 at www.scimagojr.com. Prospective, retrospective and clinical trials were included 2562. A total
\end{abstract}

\footnotetext{
Facultad de Odontología, Institución Universitaria Colegios de Colombia UNICOC. Bogotá, Colombia.

Posgrado en Periodoncia, Facultad de Odontología, Institución Universitaria Colegios de Colombia UNICOC. Bogotá, Colombia.

3 Posgrado en Periodoncia, Facultad de Odontología, Universidad Antonio Nariño. Bogotá, Colombia.
} 
of 2562 prospective, retrospective and clinical trials were included. After analyzing the titles and reading the abstracts and full texts, 6 articles were selected for analysis of the surgical technique and 35 to complement information. Conclusions: The placement of dental implants simultaneously with maxillary sinus elevation is one of the techniques used to restore function in the posterior maxilla. The most frequent intra-operative complication is perforation of the sinus membrane, and the most common post-operative complication is infection associated with perforation of the membrane, or migration of the implant to the maxillary sinus. The average implant survival reported is greater than $94 \%$.

Keywords (MeSH): Maxillary sinus; dental implants; intraoperative complications; postoperative complications

\section{INTRODUCCIÓN}

Las restauraciones implantosoportadas que se ubican en el sector posterior-superior son un reto para el clínico, debido a la presencia del seno maxilar. Este puede desarrollar una neumatización fisiológica o patológica posterior a las exodoncias de los molares, lo que impide el posicionamiento de implantes dentales $\left({ }^{1,2}\right)$.

Las técnicas de elevación de seno maxilar fueron desarrolladas por Boyne, James y Tatum (2,3). Las técnicas de ventana lateral y transalveolar permiten aumentar el tejido óseo en sentido vertical en el sector posterior $\left.{ }^{4-7}\right)$. Sin embargo, existen otras opciones ante el insuficiente tejido óseo, como el uso de implantes dentales cortos o angulados, pero estos estarían indicados solo en casos en los que existan la anatomía y las condiciones protésicas idóneas $\left({ }^{8,9}\right)$.

La literatura ha generado un debate en torno al momento en el que se debe posicionar un implante dental cuando el reborde alveolar presenta reabsorción severa $(\leq 4 \mathrm{~mm})$. En algunos estudios se realiza en dos fases $\left({ }^{10-12}\right)$ y en otros se sugiere la implantación simultánea, luego de la elevación del piso del seno maxilar $\left({ }^{13-18}\right)$, la cual es útil porque disminuye el tiempo y las etapas quirúrgicas para el paciente. Sin embargo, los resultados del procedimiento en una sola etapa dependen del operador y, por tanto, se pueden generar algunas complicaciones $\left({ }^{15}\right)$.

Por consiguiente, el objetivo de esta revisión de literatura es evaluar la supervivencia del implante dental y las complicaciones intra y posoperatorias en procedimientos de elevación del seno maxilar con técnica de ventana lateral y posicionamiento de implante dental simultáneo en rebordes con reabsorción ósea severa $(\leq 4 \mathrm{~mm})$, evaluados en un periodo de seguimiento de 2 a 96 meses tras la carga funcional.

\section{MATERIALES Y MÉTODOS}

Se realizó una búsqueda bibliográfica de la literatura publicada en los últimos 10 años durante el periodo de mayo y junio del 2019. Se desarrolló una estrategia de búsqueda en las siguientes bases de datos: MedlinePubMed, EBSCOhost y Scopus, y se complementó con una búsqueda manual en revistas especializadas en periodoncia Q1 (Periodontology 2000, Journal of Clinical Periodontology y Journal of Periodontology) y en cirugía oral Q1 (Clinical Oral Implants Research, Clinical Implant Dentistry and Related Research, European Journal of Oral Implantology), posicionadas en el top 5 del 2018 en www.scimagojr.com. Los términos MeSH utilizados fueron "maxillary sinus", "dental implant", "intraoperative complications", "postoperative complications", "bone grafting", "survival rate" y las palabras claves title/abstract fueron "lateral", "simultaneous" "sinus lift", "lateral window approach" y "primary stability".

Asimismo, se emplearon las siguientes ecuaciones de búsqueda: (()maxillary sinus[MeSH Terms]) AND (dental implant[MeSH Terms])) AND (lateral[Title/Abstract])) AND (simultaneous[Title/ Abstract]), (((sinus lift[Title/Abstract]) AND (dental implant[MeSH Terms])) AND (lateral[Title/ Abstract])) AND (simultaneous[Title/Abstract]), ((maxillary sinus[MeSH Terms]) AND (dental implant[MeSH Terms])) AND (lateral window approach[Title/Abstract]), ((dental implant[MeSH Terms]) AND (sinus lift[Title/Abstract])) AND (lateral window approach[Title/Abstract]), ((maxillary sinus[MeSH Terms]) AND (dental implant[MeSH Terms])) AND (intraoperative complications[MeSH Terms]), ((maxillary sinus[MeSH Terms]) AND (dental implant[MeSH Terms])) AND (postoperative complications $[\mathrm{MeSH} \quad$ Terms $]), \quad((($ maxillary 
sinus[MeSH Terms]) AND (dental implant[MeSH Terms])) AND (lateral[Title/Abstract])) AND (simultaneous[Title/Abstract])) AND (bone grafting[MeSH Terms]), ((maxillary sinus[MeSH Terms]) AND (dental implant[MeSH Terms])) AND (primary stability[Title/Abstract]), ((maxillary sinus[MeSH Terms]) AND (dental implant[MeSH Terms])) AND (survival rate[MeSH Terms]), (((maxillary sinus[MeSH Terms]) AND (dental implant[MeSH Terms])) AND (survival rate[MeSH Terms])) AND (bone grafting[MeSH Terms]).

Los criterios de inclusión utilizados para el análisis de la técnica quirúrgica fueron los siguientes: 1) estudios publicados entre 2009-2019; 2) ensayos clínicos prospectivos y retrospectivos en humanos; 3) estudios publicados en inglés y español, y 4) estudios en los que se evaluó la técnica de elevación del seno maxilar simultánea a la colocación de implantes en rebordes residuales $\leq 4$ $\mathrm{mm}$ en sentido ápico-coronal $\mathrm{y}$, adicionalmente, que incluyeron la variable supervivencia de los implantes, con un periodo de carga mínimo de dos meses. Los criterios de exclusión fueron los siguientes: 1) estudios que evaluaron la técnica transalveolar; 2) estudios comparativos de la técnica de elevación del seno maxilar en una o dos etapas; 3) estudios en los que se evaluó la elevación del seno maxilar simultánea a la colocación de implantes dentales, más regeneración ósea guiada horizontal o vertical.

Al finalizar la búsqueda bibliográfica, se identificaron 2562 artículos. Posteriormente al análisis de títulos, la lectura de los resúmenes y los textos completos, se seleccionaron 6 artículos para análisis de la técnica quirúrgica (tabla 1) y 35 para complemento de la información (figura 1).

\section{Elevación de seno maxilar e implante dental en rebordes con reabsorción ósea severa}

\section{Altura de reborde residual vs. altura obtenida}

La colocación de implantes dentales en la zona del maxilar superior posterior, en algunos casos, puede ser un reto por la inadecuada altura del reborde residual remanente $\left({ }^{19}\right)$, lo que genera una alteración de la estabilidad primaria del implante, el cual es un factor relevante en la osteointegración de los implantes dentales $\left({ }^{20}\right)$. En algunos estudios se recomienda el uso de una sola técnica quirúrgica o combinarlas. La elección de la técnica estará ligada a la altura residual remanente. Además, los autores han sugerido una clasificación para la elección de la técnica según su experiencia clínica $\left({ }^{21,22}\right)$. Los estudios evaluados en la actual revisión (13-18) (tabla 1) demuestran que puede existir osteointegración en rebordes con reabsorción ósea severa $(\leq 4 \mathrm{~mm})$ y evidencian que, tras el procedimiento quirúrgico, la altura del reborde se recupera en promedio unos $12,7 \mathrm{~mm}$. Por lo tanto, se considera que esta técnica quirúrgica sí logra recuperar el tejido óseo perdido por la neumatización del seno maxilar.

\section{Ancho del reborde alveolar residual al momento de la elevación de seno maxilar}

Debido al déficit en la altura del reborde residual en zona del maxilar superior posterior, que impide la colocación del implante en el mismo acto quirúrgico de la elevación del seno maxilar y su correcta estabilidad primaria, es necesario que el reborde residual cuente con un ancho adecuado $\left({ }^{13}\right)$. Los estudios evaluados (tabla 1) demuestran que se requiere una medida de $>5 \mathrm{~mm}$ para obtener tejido óseo remanente en la zona vestibular y palatina del implante dental, y que no sea necesario realizar una regeneración ósea guiada adicional (13-18).

\section{Torque de inserción y diámetro de los implantes dentales}

El torque de inserción (TI) es una variable de fácil acceso y la más utilizada para evaluar la estabilidad primaria del implante en el momento quirúrgico $\left({ }^{20}\right)$. La estabilidad primaria fue definida por Monje et al. como la traba mecánica alcanzada en el momento del posicionamiento del implante y está determinada por factores como la longitud, el diámetro y el macrodiseño del implante (diámetro/longitud y diseño de la rosca), el protocolo de preparación ósea para el implante y la macroarquitectura ósea $\left.{ }^{23}\right)$. 
Tabla 1. Estudios incluidos para análisis de la técnica quirúrgica (complicaciones, injerto óseo, supervivencia o éxito del implante)

\begin{tabular}{|c|c|c|c|c|c|c|c|c|c|c|c|c|c|}
\hline Estudio & 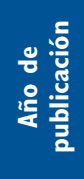 & $\begin{array}{l}\text { Diseño del } \\
\text { estudio }\end{array}$ & Institución & 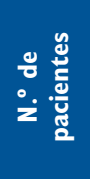 & 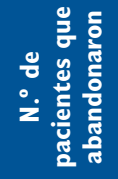 & $\begin{array}{l}\text { Rango } \\
\text { de } \\
\text { edad }\end{array}$ & $\begin{array}{l}\text { Media } \\
\text { de } \\
\text { edad }\end{array}$ & 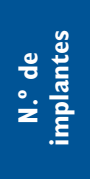 & $\begin{array}{l}\text { Ancho } \\
\text { hueso } \\
\text { residual }\end{array}$ & $\begin{array}{l}\text { Altura } \\
\text { hueso } \\
\text { residual }\end{array}$ & $\begin{array}{l}\text { Profilaxis } \\
\text { antibiótica }\end{array}$ & $\begin{array}{l}\text { Membrana } \\
\text { para cubrir } \\
\text { la ventana }\end{array}$ & $\begin{array}{l}\text { Material de } \\
\text { injerto óseo }\end{array}$ \\
\hline Chaushu et al. & 2009 & Prospectivo & Universidad & 28 & Ninguno & $25-65$ & 54,9 & 72 & $6,3 \mathrm{~mm}$ & I a $4 \mathrm{~mm}$ & $\begin{array}{l}\text { Amoxicilina } \\
1000 \text { mg antes } \\
\text { de la cirugia }\end{array}$ & Reabsorbible & $\begin{array}{c}\text { Aloinjerto } \\
\text { Bloque cancelar }\end{array}$ \\
\hline
\end{tabular}

\begin{tabular}{|c|c|c|c|c|c|c|c|c|c|c|c|c|c|}
\hline & & & & & & & & & & & Clindamicina & & \\
\hline Manso et al. & 2010 & Prospectivo & Universidad & 45 & Ninguno & $26-80$ & 54 & 160 & NR & $\leq 4 \mathrm{~mm}$ & $\begin{array}{c}300 \text { mg, I hora } \\
\text { antes de la } \\
\text { cirugía }\end{array}$ & Reabsorbible & $\begin{array}{c}\text { Autoinjerto y } \\
\text { aloplástico }\end{array}$ \\
\hline
\end{tabular}

\begin{tabular}{|c|c|c|c|c|c|c|c|c|c|c|c|c|c|}
\hline Canullo et al. & 2010 & Prospectivo & Universidad & 30 & Ninguno & $>18$ & 58,3 & 67 & $4-5 \mathrm{~mm}$ & I-4 mm & $\begin{array}{c}\text { Ig de } \\
\text { clavulonato; } \\
450 \text { mg de } \\
\text { clindamicina } \\
\text { antes de la } \\
\text { cirugía }\end{array}$ & No usaron & Aloplástico \\
\hline
\end{tabular}

\begin{tabular}{|c|c|c|c|c|c|c|c|c|c|c|c|c|c|}
\hline Merli et al. & 2013 & Ensayo clínico & $\begin{array}{l}\text { Práctica } \\
\text { privada }\end{array}$ & 40 & I & $>18$ & NR & 59 & NR & $1-3 \mathrm{~mm}$ & NR & Reabsorbible & $\begin{array}{l}\text { Autoinjerto vs. } \\
\text { xenoinjerto }\end{array}$ \\
\hline
\end{tabular}

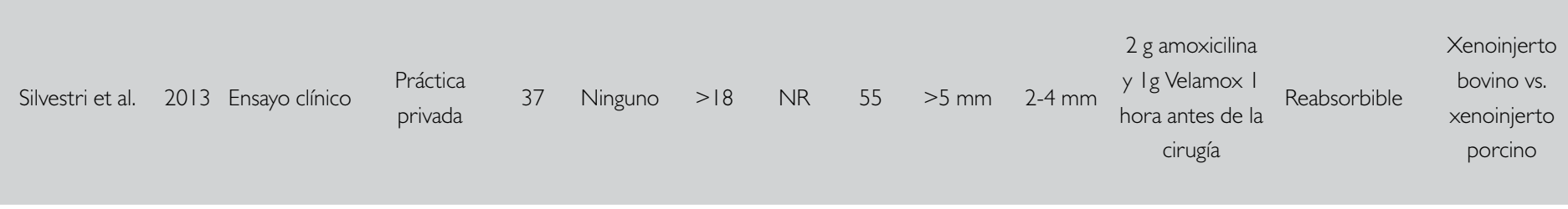

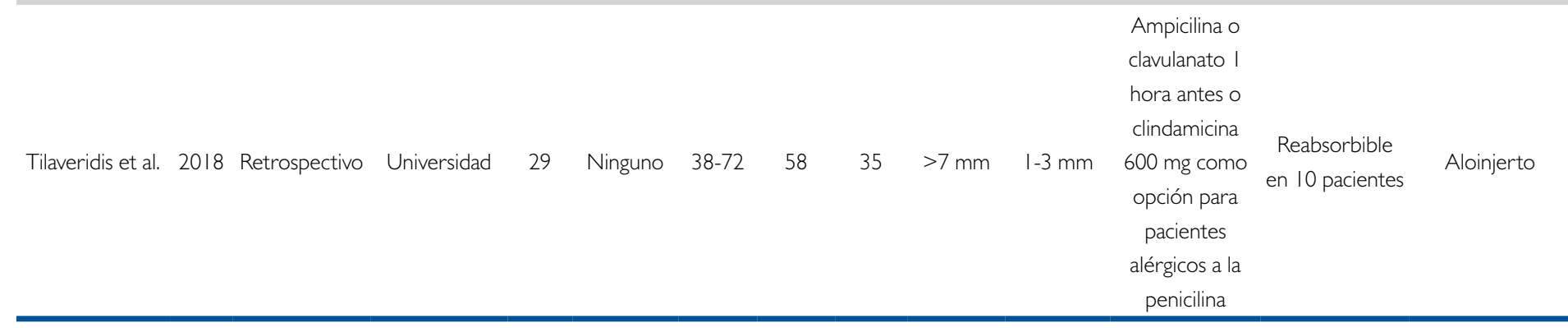




\begin{tabular}{|c|c|c|c|c|c|c|c|c|c|c|}
\hline \multicolumn{5}{|c|}{ Procedimiento quirúrgico } & \multirow[b]{2}{*}{$\begin{array}{l}\text { Medicación } \\
\text { posoperatoria }\end{array}$} & \multirow{2}{*}{ 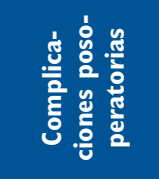 } & \multirow[b]{2}{*}{$\begin{array}{l}\text { Altura hueso } \\
\text { posterior al } \\
\text { procedimiento }\end{array}$} & \multirow{2}{*}{$\begin{array}{l}\text { Tiempo } \\
\text { para la } \\
\text { segunda } \\
\text { fase } \\
\text { quirúrgica }\end{array}$} & \multirow[b]{2}{*}{$\begin{array}{l}\text { Seguimiento } \\
\text { posterior } \\
\text { de la carga }\end{array}$} & \multirow[b]{2}{*}{$\begin{array}{l}\text { Supervivencia } \\
\text { de implantes }\end{array}$} \\
\hline $\begin{array}{l}\text { Marca de } \\
\text { implantes }\end{array}$ & $\begin{array}{l}\text { Diámetro } \\
\text { de } \\
\text { implantes }\end{array}$ & $\begin{array}{l}\text { Longitud } \\
\text { de } \\
\text { implantes }\end{array}$ & $\begin{array}{l}\text { Torque de } \\
\text { inserción }\end{array}$ & Complicaciones & & & & & & \\
\hline $\begin{array}{c}\text { Miss, Zimmer } \\
\text { y } 3 i\end{array}$ & $\begin{array}{l}3,7 \mathrm{~mm} \\
4,0 \mathrm{~mm} \\
4,2 \mathrm{~mm}\end{array}$ & $13 \mathrm{~mm}$ & $\begin{array}{c}\text { Se consiguió } \\
\text { estabilidad } \\
\text { primaria, } \\
\text { pero no } \\
\text { reporta } \\
\text { torque. }\end{array}$ & $\begin{array}{l}\text { Perforaciones } \\
\qquad 21,4 \%\end{array}$ & $\begin{array}{l}\text { Amoxicilina } 500 \text { mg, } \\
3 \text { veces al día por } \\
10 \text { días; etodolaco } \\
600 \text { mg, } 2 \text { veces al } \\
\text { día; clorhexidina } \\
0,2 \%, 2 \text { veces al día } \\
\text { por } 2 \text { semanas }\end{array}$ & Ninguna & $12,3 \mathrm{~mm}$ & 4 meses & I I-46 meses & $94,4 \%$ éxito \\
\hline Nobel, $3 i$ & NR & NR & $\begin{array}{c}\text { Se } \\
\text { consiguió } \\
\text { estabilidad } \\
\text { primaria, } \\
\text { pero no } \\
\text { reporta } \\
\text { Torque. }\end{array}$ & $\begin{array}{c}\text { Perforaciones } \\
8,7 \%\end{array}$ & $\begin{array}{c}\text { Clindamicina } 300 \\
\text { mg, } 3 \text { veces al } \\
\text { día por I } 4 \text { días; o } \\
\text { clavulin, } 3 \text { veces } \\
\text { al día por I } 4 \text { días; } \\
\text { acetaminofén/ } \\
\text { lbuprofeno y } \\
\text { dexametasona, } 72 \\
\text { horas después de la } \\
\text { cirugía }\end{array}$ & $\begin{array}{c}\text { I Infección } \\
\text { moderada } \\
2 \text { semanas } \\
\text { después de la } \\
\text { cirugía }\end{array}$ & NR & II meses & 6 meses & $\begin{array}{c}\text { 94,85\% éxito; } \\
98,05 \% \\
\text { supervivencia }\end{array}$ \\
\hline
\end{tabular}

\begin{tabular}{|c|c|c|c|c|c|c|c|c|c|c|}
\hline Martina & $\begin{array}{l}4,3 \mathrm{~mm} \\
4,8 \mathrm{~mm} \\
5,5 \mathrm{~mm}\end{array}$ & $13 \mathrm{~mm}$ & $>10 \mathrm{~N}$ & $\begin{array}{c}\text { Perforaciones en } \\
4 \text { casos }\end{array}$ & $\begin{array}{c}2 \mathrm{~g} \text { de clavulonato } \\
\text { o } 450 \mathrm{mg} \text { de } \\
\text { clindamicina por } \\
6 \text { días }\end{array}$ & $\begin{array}{c}\text { Sangrado nasal } \\
\text { leve en un } \\
\text { caso }\end{array}$ & $\begin{array}{l}\text { 13,75 mm: } 24 \\
\text { meses después }\end{array}$ & 3 meses & 24 meses & $\begin{array}{c}97 \% \\
\text { Supervivencia }\end{array}$ \\
\hline
\end{tabular}

\section{Ibruprofeno 600}

$\mathrm{mg}, 2$ veces al

día por 3 días;

Perforaciones clorhexidina $0,12 \%$ Periimplantitis

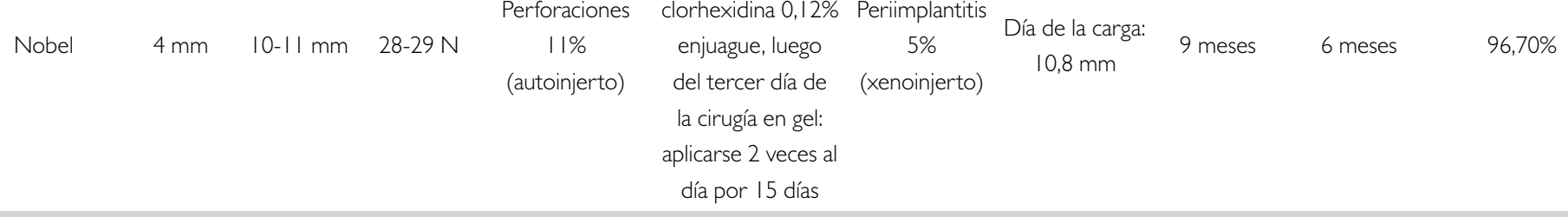

\begin{tabular}{|c|c|c|c|c|c|c|c|c|c|c|}
\hline Thomen & $4-4,5 \mathrm{~mm}$ & $\begin{array}{c}9,5-1 \mid, 5 \\
\mathrm{~mm}\end{array}$ & I I,5-34,3 N & $\begin{array}{c}\operatorname{Sin} \\
\text { complicaciones }\end{array}$ & $\begin{array}{l}\text { velamox, } 2 \text { veces al } \\
\text { día por una semana; } \\
\text { nimesulida } 100 \text { mg, } \\
2 \text { veces al día por } \\
3 \text { días }\end{array}$ & Ninguna & NR & 6 meses & 2 meses & $96,34 \%$ \\
\hline
\end{tabular}

Se consiguió

estabilidad

C-Tech $\quad 3,5-4,5 \mathrm{~mm} \quad 13 \mathrm{~mm} \quad$ primaria,

pero no

reporta

torque
Día de la $\begin{array}{cc}\text { posoperatoria } & \text { cirugía: } 15 \mathrm{~mm} \\ \text { I año después: }\end{array}$ migró al seno
maxilar $\quad \begin{aligned} & 12 \mathrm{~mm} \\ & \text { años después: }\end{aligned}$ $11 \mathrm{~mm}$ 
Monje et al. demostraron que no hay diferencia estadísticamente significativa en la supervivencia de implantes que han sido colocados a diferentes torques de inserción ( $5 \mathrm{Ncm}$ a $70 \mathrm{Ncm}$ ) con un valor $\mathrm{p}=$ 0,227 (23). Sin embargo, en los estudios evaluados en la presente revisión ( ${ }^{13-18}$ ) (tabla 1) sí se recomienda lograr la estabilidad primaria, a través de lo siguiente: 1) Seguir el protocolo de fresado de la casa comercial para hueso tipo III o IV, es decir, preparar hasta 1 o 2 fresas antes de la final; y 2) En caso de no lograr estabilidad primaria, cambiar el implante a uno de mayor diámetro.

Es natural pensar que un mayor TI asegurará la osteointegración de los implantes dentales y que un TI
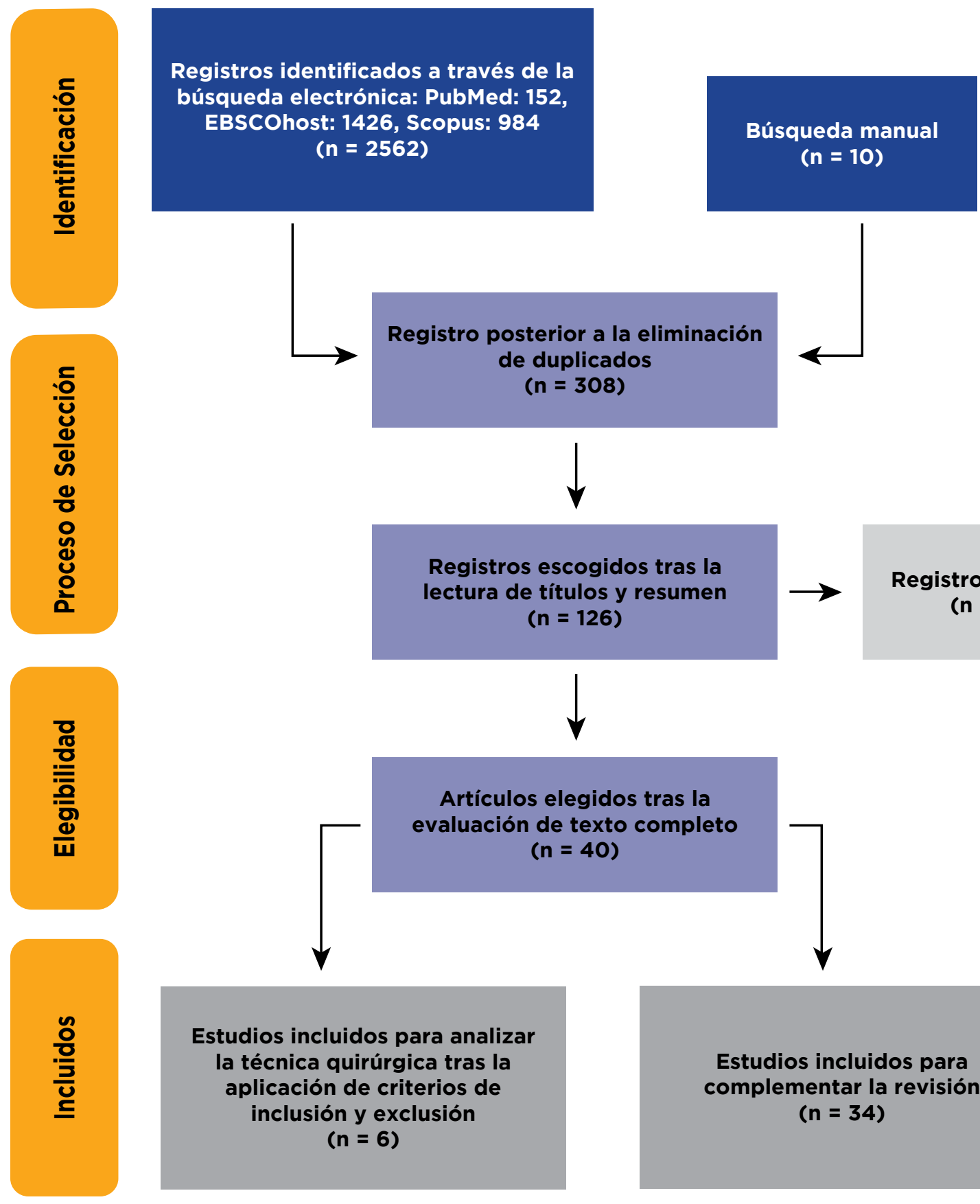

Registro posterior a la eliminación de duplicados

( $n=308)$
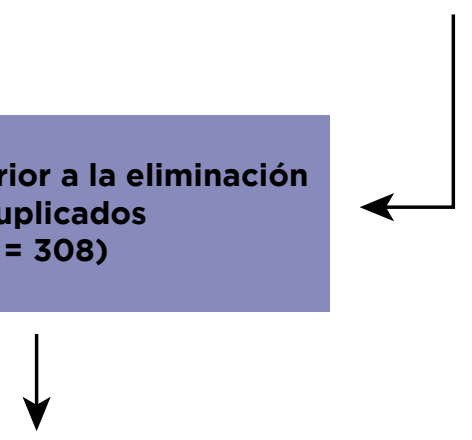

Registros escogidos tras la lectura de títulos y resumen $(n=126)$

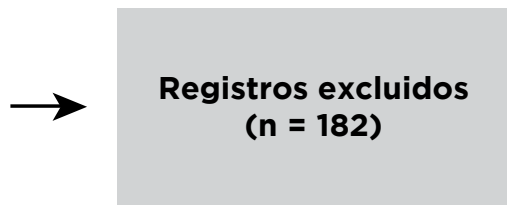

Artículos elegidos tras la evaluación de texto completo $(n=40)$

Estudios incluidos para analizar la técnica quirúrgica tras la aplicación de criterios de inclusión y exclusión $(n=6)$

Estudios incluidos para complementar la revisión $(n=34)$ 
bajo puede conducir a una menor estabilidad del implante y causar una posible fibrointegración. Sin embargo, la literatura demuestra que un $\mathrm{TI}>50 \mathrm{Ncm}$ obtenido por subpreparación puede producir microfracturas óseas que impiden el proceso de osteointegración. Además, un TI menor no afecta este proceso siempre que no se vea interrumpido por micromovimientos $\left({ }^{23}\right)$.

Los estudios evaluados (tabla 1) reportan un TI entre $11 \mathrm{Ncm}$ y $34 \mathrm{Ncm}\left({ }^{13-18}\right)$. Además, se observó que el diámetro promedio de los implantes fue de $4 \mathrm{~mm}$, con un rango entre 3,5 y 4,5 $\mathrm{mm}$. Este diámetro permitió la conservación de la tabla ósea vestibular y palatina de 1,5 $\mathrm{mm}$, con el fin de evitar la reabsorción del tejido óseo remanente durante el remodelado óseo y recesiones por dehiscencias o fenestraciones.

\section{Injerto óseo}

En las últimas décadas, se han desarrollado diferentes tipos de injertos óseos. El estándar de oro en procedimientos regenerativos sigue siendo el injerto óseo autólogo. Es considerado confiable y seguro. Se puede obtener de diferentes zonas, tanto intra como extraorales, aunque se prefieren las intraorales por su accesibilidad. Sin embargo, este tipo de injerto requiere de otra zona quirúrgica, lo que genera mayor morbilidad en el paciente $\left({ }^{24}\right)$. Para evitar este procedimiento adicional, se puede utilizar otro tipo de injertos óseos como los aloinjertos, xenoinjertos y materiales aloplásticos. Los implantes posicionados en zonas injertadas con este tipo de materiales han demostrado resultados similares en osteointegración y supervivencia al compararlos con sitios injertados con hueso autólogo $\left.{ }^{25}\right)$. Asimismo, se ha observado que se puede producir una regeneración espontánea al permitir la formación del coágulo sanguíneo en sitios donde se realice la elevación de seno maxilar, sin ningún tipo de injerto óseo $\left.{ }^{26}\right)$.

En los procedimientos de elevación del seno maxilar con técnica de ventana lateral en rebordes con reabsorción ósea severa $(\leq 4 \mathrm{~mm})$ y colocación simultánea de implantes dentales, luego de verificar la estabilidad primaria del implante, se rellena el defecto con el material de injerto óseo elegido según el caso. En la presente revisión (tabla 1), los estudios que compararon el autoinjerto con el xenoinjerto, se observó una tasa de supervivencia de los implantes mayor al $96 \%$ en un periodo de seguimiento de 6 meses posterior a la carga, sin diferencias estadísticamente significativas entre ambos injertos $(\mathrm{p}=0,9283)\left({ }^{13}\right)$. Al comparar el uso de xenoinjerto bovino con xenoinjerto porcino, tampoco se encontraron diferencias estadísticamente significativas al evaluar el volumen total óseo $(p=0,99)$ y la formación de tejido osteoide $(p=0,64)$ en un periodo de seguimiento de 2 meses posterior a la carga $\left({ }^{14}\right)$.

Por otro lado, en un estudio prospectivo que utilizó la combinación de aloplástico con autoinjerto se observó una tasa de éxito del 94,85\% en un periodo de seguimiento de 6 meses posterior a la carga $\left({ }^{18}\right)$. En los estudios prospectivos y retrospectivos que solo utilizaron un material de injerto como el aloinjerto en bloque, se observó una tasa de éxito mayor al $94 \%$ en un periodo de seguimiento de 11 a 46 meses posteriormente a la carga. Para el aloinjerto particulado, se observó una tasa de supervivencia de los implantes mayor al $94 \%$ en un periodo de seguimiento de 3 a 8 años posteriormente a la carga, y para el aloplástico se observó una tasa de supervivencia de los implantes del $97 \%$ en un periodo de seguimiento de 24 meses tras la carga $\left({ }^{15-17}\right)$.

\section{Complicaciones intraoperatorias}

Las complicaciones intraoperatorias (CI) se definen como la desviación inesperada del curso del tratamiento normal durante el procedimiento quirúrgico $\left({ }^{13}\right)$. Las CI descritas en la literatura con mayor frecuencia de aparición son la perforación de la membrana de Schneider, en un 23,5\% (con un rango entre el 3,6\% y el $41,8 \%)$, y las complicaciones hemorrágicas, en un $0,4 \%$ $\left({ }^{27,} 28\right)$. De igual forma, en los estudios analizados en la presente revisión (tabla 1), se reportó la perforación de la membrana de Schneider, en un rango del 8\% al 21,4\%,y no se registró ninguna complicación hemorrágica $\left({ }^{13,15-18}\right)$.

Stacchi et al. y Geminiani et al. estudiaron la relación entre el uso de diferentes equipos para realizar la ventana lateral (pieza de alta, pieza de baja, raspadores, sónicos, ultrasónicos) y la incidencia de la perforación 
de la membrana de Schneider $\left({ }^{28,29}\right)$. Stacchi et al. no encontraron ninguna relación $\left.{ }^{(29}\right)$, mientras que Geminiani et al. observaron que el uso de equipos ultrasónicos tiene un riesgo de perforación del 10,9\% y los equipos rotatorios, del 20,1\% (28). Otros factores que pueden aumentar el riesgo de perforación son el espesor reducido de la membrana y la presencia de tabiques sinusales $\left({ }^{27}\right)$. No existe diferencia estadísticamente significativa que demuestre la influencia negativa de la perforación de la membrana con el riesgo de falla en la osteointegración de los implantes, al compararlo con membranas intactas $(p=0,24)\left({ }^{27}\right)$.

Las perforaciones de la membrana se pueden manejar en el mismo tiempo quirúrgico posicionando una membrana de colágeno que cubra el sitio de la perforación $(13,15,16,27)$, con una membrana de fibrina o suturando la perforación $\left.{ }^{(29}\right)$. Si esta perforación no se trata de forma adecuada, puede imposibilitar la estabilización del material de injerto óseo particulado o su diseminación en la cavidad sinusal de forma inmediata o tardía $\left({ }^{30}\right)$. Al Dajani et al. recomiendan el posicionamiento del implante simultáneo a la elevación del seno cuando la perforación no sobrepasa los $6 \mathrm{~mm}$ de longitud $\left({ }^{27}\right)$. Sin embargo, no especifican en qué casos debe darse por inviable terminar el procedimiento. En los estudios evaluados (tabla 1), en algunos casos, se reportó una perforación de la membrana de tamaño pequeño, sin afectación de la osteointegración o el injerto óseo.

Las complicaciones hemorrágicas se presentan al lacerar los vasos responsables del suministro sanguíneo del seno maxilar, como las arterias infraorbitarias, palatinas mayores, alveolares posteriores superiores y sus ramas intraóseas y anastomosis ${ }^{31}$. Específicamente, la anastomosis entre la arteria alveolar-antral y la infraorbitaria ubicada en la pared lateral del seno maxilar es susceptible a daños accidentales durante la conformación de la ventana lateral $\left({ }^{31}, 32\right)$. La correcta evaluación tomográfica preoperatoria permite ubicar su localización en el 63,36\% de los casos. Su diámetro promedio es de $<1 \mathrm{~mm}$, con un rango entre 1 y $2 \mathrm{~mm}$, y se ubica entre $11,25 \mathrm{~mm}$ y $26,90 \mathrm{~mm}$ desde la cresta residual al piso del seno maxilar ${ }^{(32}$. La hemorragia puede generar dificultad en la visibilidad del campo quirúrgico; por tanto, debe ser tratada adecuadamente con electrocauterio, cera ósea, compresión con gasas estériles embebidas en solución salina o ácido tranexámico. Es importante controlarla para finalizar el procedimiento y evitar así un sangrado secundario posoperatorio y hematomas varias horas después de finalizada la cirugía $\left({ }^{28,33}\right)$. Sin embargo, los eventos hemorrágicos representan una complicación extremadamente infrecuente $\left({ }^{27,31}\right)$.

\section{Complicaciones posoperatorias}

Al igual que las $\mathrm{CI}$, las complicaciones posoperatorias (CP) se pueden presentar y afectar el éxito a corto o largo plazo de los implantes dentales. Sin embargo, si se ejecuta un protocolo adecuado, se puede continuar el seguimiento respectivo sin ningún problema. Las $\mathrm{CP}$ pueden ser leves o severas, y biológicas o protésicas. Las que se reportan con mayor frecuencia en la literatura evaluada se describen a continuación.

\section{Consecuencias posoperatorias de la perforación de la membrana sinusal}

El tamaño de la perforación de la membrana juega un factor determinante en la supervivencia de los implantes dentales $\left({ }^{27}\right)$. Estudios demuestran que, entre más grande sea la perforación, disminuye la supervivencia y estabilidad de los implantes y la formación del tejido óseo $(p=<0,0001)\left({ }^{33}\right)$, en comparación con membranas intactas. La presencia de perforaciones puede aumentar hasta 3 veces el riesgo de fallo del injerto óseo $(\mathrm{p}=0,003)$ y hasta 6 veces el riesgo de infección o sinusitis $(\mathrm{p}=<0,0006)\left({ }^{34}\right)$. Sin embargo, si la perforación se maneja de forma adecuada, se pueden lograr resultados exitosos y evitar complicaciones mayores $\left({ }^{27}\right)$.

\section{Infección}

Es una de las complicaciones de mayor cuidado, sin subestimar las anteriores, ya que puede requerir incisión y drenaje, y desencadenar la pérdida parcial o total del injerto óseo o del implante dental. Además, si no se trata a tiempo, puede propagarse como una infección sistémica y traer consecuencias irreversibles para el paciente $\left({ }^{35}\right)$. Kozuma et al. definen la infección posoperatoria como 
una descarga purulenta o una inflamación supurativa en el sitio quirúrgico $\left.{ }^{(35}\right)$. Pero esta complicación no es frecuente. La literatura reporta una incidencia del 1\% al $12 \%\left({ }^{36}\right)$, información que coincide con lo observado en los estudios evaluados (tabla 1) ${ }^{16,18}$ ), en los que se presentó esta complicación en tan solo dos pacientes. La infección se resolvió posteriormente al tratamiento prolongado con antibioticoterapia y los implantes dentales no fallaron.

Los pacientes con infección pueden presentar signos y síntomas desde la segunda a la quinta semana luego del procedimiento quirúrgico $\left({ }^{37}\right)$. Entre los signos y síntomas se encuentran la congestión nasal, la presión sinusal, el dolor de cabeza, el vértigo, el drenaje nasal purulento, la fístula intraoral y el edema intraoral o extraoral. Esto puede desencadenar una sinusitis aguda o rinosinusitis $\left({ }^{35,38}\right)$, y en el caso de no ser tratadas a tiempo se podría desarrollar un absceso cerebral que requiere manejo hospitalario $\left.{ }^{38}\right)$. Sin embargo, esta infección se puede prevenir si se evalúa cada caso con cautela, mediante la revisión de los antecedentes sistémicos y locales, así como un correcto manejo quirúrgico y posquirúrgico. Kozuma et al. encontraron una relación positiva entre la sinusitis crónica diagnosticada de manera prequirúrgica y la infección posoperatoria $(\mathrm{p}=0,007)\left({ }^{35}\right)$.

Por lo anteriormente mencionado, según el grado de infección y los síntomas que presente el paciente, algunos estudios sugieren de manera inicial el uso de antibióticos por vía oral y la reevaluación. Si no evoluciona de forma positiva, se debe tratar con abordaje quirúrgico para remover de forma parcial o total el injerto óseo, el tejido granulomatoso o el implante dental, limpiar la cavidad e injertar o no, según la evaluación individual cada caso $(35,37,38)$.

\section{Migración de implantes al seno maxilar}

La migración del implante al seno maxilar ocurre comúnmente durante la colocación del implante o se identifica durante la segunda fase quirúrgica, y en algunos casos aislados se puede presentar luego de la carga $\left({ }^{39}\right)$. Esta complicación está asociada a la inadecuada altura ósea, la presencia de hueso tipo IV, la insuficiente estabilidad primaria o una reabsorción ósea severa debido a una infección tipo periimplantitis, mala planificación quirúrgica, técnica quirúrgica inadecuada y baja experiencia del operador $\left({ }^{39,}{ }^{40}\right)$. Galindo et al. proponen tres causas probables de fracaso: cambios en las presiones de aire intrasinusal, lo cual produce un efecto de succión por la presión negativa; reacción autoinmune al implante, que produce destrucción ósea periimplantar y compromete la osteointegración; y reabsorción ósea producida por una distribución incorrecta de las fuerzas oclusales $\left({ }^{41}\right)$.

Los signos y síntomas presentados incluyen sinusitis (67\%), fístula y comunicación oroantral (83\%), goteo de sangre o material purulento por las fosas nasales (49\%), obstrucción nasal (23\%) y dolor $(16 \%)\left({ }^{40}\right)$. Por lo tanto, los cuerpos extraños en el seno maxilar deben retirarse lo antes posible y la comunicación oroantral debe cerrarse con colgajos para evitar tales complicaciones $\left({ }^{40}\right)$. En la actualidad, existen dos modalidades de tratamiento principales para la extracción del implante desplazado al seno maxilar y el tratamiento de las complicaciones infecciosas asociadas: abordaje intraoral por medio de una ventana ósea en la pared lateral del seno maxilar (más frecuente) y abordaje transnasal con cirugía sinusal endoscópica funcional $\left({ }^{40}\right)$. Sin embargo, esta complicación no es frecuente y solo está descrita en la literatura por reportes y series de casos $\left(^{39-41}\right)$. Esta información coincide con lo observado en los estudios evaluados (tabla 1), en los que solo un implante migró al seno maxilar en el posoperatorio. El implante fue extraído y no se presentaron signos de infección, por lo cual se solucionó con la colocación de otro implante dental en una zona distal $\left({ }^{16}\right)$.

\section{Tiempo a la segunda fase quirúrgica}

La segunda fase es de vital importancia por ser el momento quirúrgico en el que se puede verificar la osteointegración del implante y la estabilidad de los tejidos periimplantares. Estas características se logran a partir de la conexión entre la estabilidad primaria y secundaria. Monje et al. definen la estabilidad secundaria como la aposición ósea alrededor del implante que se produce tras la inserción de este, mediante la homeostasis 
de la osteointegración, influenciada por factores como el microdiseño del implante, la macro y microarquitectura ósea, y la carga del implante (23). En los estudios evaluados (tabla 1) se observó un promedio de espera desde el posicionamiento del implante de 6 meses, con un rango de 3 a 11 meses para realizar la segunda fase ${ }^{(13-18)}$. Chaushu et al. y Tilavideris et al., recomiendan tomar una radiografía previa al procedimiento con el fin de observar alrededor del implante zonas radiopacas compatibles con una masa ósea homogénea $\left({ }^{15,16}\right)$ y evaluar la estabilidad del implante al ser descubierto, sometiéndolo a torque reverso de $35 \mathrm{Ncm}\left({ }^{16}\right)$.

\section{Relación entre la falla de implantes dentales y el reborde residual inicial}

El reborde residual, como ya se mencionó anteriormente, es de vital importancia para la estabilidad primaria de los implantes dentales y más en la técnica quirúrgica de elevación del seno maxilar con colocación simultánea de implante dental. Manor et al. evaluaron las posibles causas de la migración del implante dental al seno maxilar y encontraron que, de los 55 casos evaluados, en 37 la altura de hueso estaba en un rango de 0-5 mm $\left({ }^{40}\right)$. Pese a esto, en los estudios evaluados (tabla 1), el promedio de supervivencia de los implantes dentales colocados de manera simultánea a la elevación del seno maxilar en rebordes con reabsorción ósea severa $(\leq 4 \mathrm{~mm})$ fue del 95\%. E1 5\% restante corresponde a implantes que no se osteointegraron o que fracasaron durante la carga, pero no se relacionó la falla con la altura ósea residual previa $\left.{ }^{13-18}\right)$. Tilaveridis et al. describen la medida exacta de la altura del reborde óseo en los implantes con complicaciones y evalúan un total de 35 implantes, de los cuales solo 2 presentaron complicaciones en un reborde de $1 \mathrm{~mm}$ de altura. Sin embargo, 8 implantes posicionados a la misma altura no presentaron ningún tipo de complicación, con un periodo de seguimiento de 5 años $\left({ }^{16}\right)$.

\section{CONCLUSIONES}

La elevación del seno maxilar con técnica de ventana lateral e inserción simultánea de implantes dentales en reborde óseo con reabsorción severa $(\leq 4 \mathrm{~mm})$ es una técnica utilizada para restituir la función en el maxilar posterior que ha demostrado una supervivencia promedio en los estudios evaluados mayor al $94 \%$, con un seguimiento de 2 a 96 meses poscarga, lo que la posiciona como una opción terapéutica. Sin embargo, esta técnica no está exenta de complicaciones. La complicación intraoperatoria más frecuente es la perforación de la membrana sinusal y la posoperatoria es el riesgo de infección asociada con la perforación de la membrana o la migración del implante al seno maxilar.

Contribución de autoría: Fabio Andrés Jiménez Castellanos ha sido el líder del estudio, contribuyendo con la búsqueda electrónica y revisión inicial. Paola Castro contribuyó con la búsqueda manual y la elaboración de la tabla. Christian Andrés Huertas Torres contribuyó con la búsqueda manual y redacción. Asterlis Buitrago Osuna ha sido colíder del estudio y contribuido con la redacción y revisión final.

Fuentes de financiamiento: Autofinanciada.

Conflicto de intereses: Ninguna. 


\section{REFERENCIAS BIBLIOGRÁFICAS}

1. Khoury F, Hanser T. Three-dimensional vertical alveolar ridge augmentation in the posterior maxilla: a 10 -year clinical study. Int J Oral Max Impl. 2019; 34 (2): 471-80. doi: 10.11607/ jomi.6869

2. Boyne PJ, James RA. Grafting of the maxillary sinus floor with autogenous marrow and bone. J Oral Surg. 1980; 38 (8): 613-6.

3. Tatum H Jr. Maxillary and sinus implant reconstructions. Dent Clin N Am. 1986; 30 (2): 207-29.

4. Falah M, Sohn D, Srouji S. Graftless sinus augmentation with simultaneous dental implant placement: clinical results and biological perspectives. Int J Oral Maxillofac Surg. 2016; 45 (9): 1147-53. doi: 10.1016/j.ijom.2016.05.006

5. Fouad W, Osman A, Atef M, Hakam M. Guided maxillary sinus floor elevation using deproteinized bovine bone versus graftless Schneiderian membrane elevation with simultaneous implant placement: Randomized clinical trial. Clin Implant Dent Relat Res. 2018; 20 (3): 424-33. doi: 10.1111/cid.12601

6. Summers RB. A new concept in maxillary implant surgery: the osteotome technique. Compendium. 1994; 15 (2): 152.

7. Aludden H, Mordenfeld A, Hallman M, Christensen A, Starch T. Osteotome-mediated sinus floor elevation with or without a grafting material: a systematic review and meta-analysis of longterm studies ( $\geq 5$-years). Implant Dent. 2018; 27 (4): 488-97. doi: 10.1097/ID.0000000000000798

8. Bechara S, Kubilius R, Veronesi G, Pires J, Shibli J, Mangano F. Short (6-mm) dental implants versus sinus floor elevation and placement of longer $(\geq 10-\mathrm{mm})$ dental implants: a randomized controlled trial with a 3-year follow-up. Clin Oral Implants Res. 2017; 28 (9): 1097-107. doi: 10.1111/clr.12923

9. Davó R, Felice P, Pistilli R, Barausse C, Marti C, Ferrer A, et al. Immediately loaded zygomatic implants vs conventional dental implants in augmented atrophic maxillae: 1 -year post-loading results from a multicentre randomised controlled trial. Eur J Oral Implantol. 2018; 11 (2): 145-61.

10. Krennmair G, Krainhöfner M, Schmid-Schwap M, Piehslinger E. Maxillary sinus lift for single implant-supported restorations: a clinical study. Int J Oral Maxillofac Implants. 2007; 22 (3): 351-8.

11. Baccar MN, Laure B, Chabut A, Bonin B, Romieux G, Goga D. Stabilité du greffon et des implants après greffe osseuse du sinus maxillaire [Stability of grafts and implants after bone grafting of the maxillary sinus. Retrospective analysis of 44 patients]. Rev Stomatol Chir Maxillofac. 2005; 106 (3): 153-6.

12. Ewers R. Maxilla sinus grafting with marine algae derived bone forming material: a clinical report of long-term results. J Oral Maxillofac Surg. 2005; 63 (12): 1712-23. doi: 10.1016/j. joms.2005.08.020

13. Merli M, Moscatelli M, Mariotti G, Rotundo R, Nieri M. Autogenous bone versus deproteinised bovine bone matrix in 1-stage lateral sinus floor elevation in the severely atrophied maxilla: a randomised controlled trial. Eur J Oral Implantol. 2013; 6 (1): 27-37.
14. Silvestri M, Martegani P, D'Avenia F, Farneti M, Capri D, Paolantoni $G$ et al. Simultaneous sinus augmentation with implant placement: histomorphometric $y$ comparison of two different grafting materials. A multicenter double-blind prospective randomized controlled clinical trial. Int J Oral Max Impl. 2013; 28 (2): 543-9.

15. Chaushu G, Mardinger O, Calderon S, Moses O, Nissan J. The use of cancellous block allograft for sinus floor augmentation with simultaneous implant placement in the posterior atrophic maxilla. J Periodontol. 2009; 80 (3): 422-8 doi: 10.1902/ jop.2009.080451

16. Tilaveridis I, Lazaridou M, Zouloumis L, Dimitrakopoulos I, Tilaveridis V, Tilaveridou S. The use of mineralized bone allograft as a single grafting material in maxillary sinus lifting with severely atrophied alveolar ridge (1-3 $\mathrm{mm})$ and immediately inserted dental implants. A 3- up to 8-year retrospective study. Oral Maxillofac Surg. 2018; 22 (3): 267-73. doi: 10.1007/ s10006-018-0698-6

17. Canullo L, Patacchia O, Sisti A, Heinemann F. Implant restoration 3 months after one stage sinus lift surgery in severely resorbed maxillae: 2-year results of a multicenter prospective clinical study. Clin Implant Dent Relat Res. 2012;14 :412-20. doi: 10.1111/j.1708-8208.2009.00261.x

18. Manso MC, Wassal T. A 10-years longitudinal study of 160 implants simultaneously installed in severely resorbed posterior maxillas grafted with autogenous bone and synthetic bioactive resor-bable graft. Implant Dent. 2010; 4: 351-60.

19. Thoma D, Haas R, SporniakTK, García A, Taylor TD, Hämmerle C. Randomized controlled multicentre study comparing short dental implants $(6 \mathrm{~mm})$ versus longer dental implants (11-15 $\mathrm{mm})$ in combination with sinus floor elevation procedures: 5Year. J Clin. Periodontol. 2018; 45 (12): 1465-74. doi: 10.1111/ jcpe. 13025

20. Al-Sabbagh M, Eldomiaty W, Khabbaz Y. Can osseointegration be achieved without primary stability? Dent Clin N Am. 2019; 63 (3): 461-73. doi: 10.1016/j.cden.2019.02.001

21. Wang HL, Katranji A. ABC sinus augmentation classification. Int J Periodont Rest. 2008; 28 (4): 383-9.

22. Niu L, Wang J, Yu H, Qiu L. New classification of maxillary sinus contours and its relation to sinus floor elevation surgery. Clin Implant Dent R. 2018; 20 (4): 493-500. doi: 10.1111/ cid.12606

23. Monje A, Ravidà A, Wang HL, Helms JA, Brunski JB. Relationship between primary/mechanical and secondary/ biological implant stability. Int J Oral Max Impl 2019; 34: 7-23. doi: 10.11607/jomi.19suppl.g1

24. Chavda S, Levin L. Human studies of vertical and horizontal alveolar ridge augmentation comparing different types of bone graft materials: a systematic review. J Oral Implantol. 2018; 44 (1): 74-84. doi: 10.1563/aaid-joi-D-17-00053 
25. Papageorgiou S, Papageorgiou P, Deschner J, Götz W. Comparative effectiveness of natural and synthetic bone grafts in oral and maxillofacial surgery prior to insertion of dental implants: Systematic review and network meta-analysis of parallel and cluster randomized controlled trials. J Dent. 2016; 48: 1-8. doi: 10.1016/j.jdent.2016.03.010

26. Parra M, Atala C, Fariña R, Haidar ZS, Zaror C, Olate S. Graftless maxillary sinus lift using lateral window approach: a systematic review. Implant Dent. 2018; 27 (1): 111-8. doi: 10.1097/ID.0000000000000695

27. Al-Dajani M. Incidence, risk factors, and complications of schneiderian membrane perforation in sinus lift surgery: a metaanalysis. Implant Dent. 2016; 25 (3): 409-15. doi: 10.1097/ ID.0000000000000411

28. Geminiani A, Tsigarida A, Chochlidakis K, Papaspyridakos PV, Feng C, Ercoli C. A meta-analysis of complications during sinus augmentation procedure. Quintessence Int. 2017; 48 (3): 231-40. doi: 10.3290/j.qi.a37644

29. Stacchi C, Andolsek F, Berton F, Perinetti G, Navarra C, Di Lenarda R. Intraoperative complications during sinus floor elevation with lateral approach: a systematic review. Int J Oral Max Impl. 2017; 32 (3): 107-18. doi: 10.11607/jomi.4884

30. Wallace S, Tarnow D, Froum S, Cho S, Zadeh H, Stoupel J, et al. Maxillary sinus elevation by lateral window approach: evolution of technology and technique. J Evid Based Dent Pract. 2012; 12 (3): 161-71. doi: 10.1016/S1532-3382(12)70030-1

31. Danesh S, Loomer P, Wallace S. A comprehensive clinical review of maxillary sinus floor elevation: anatomy, techniques, biomaterials and complications. Br J Oral Maxillofac Surg. 2016; 54 (7): 724-30. doi: 10.1016/j.bjoms.2016.05.008

32. Quispe D, Valdivia E, Mendoza-Azpur G, Salinas Prieto E. Evaluación de la arteria alveolar superior posterior durante el levantamiento del seno maxilar con uso de la tomografía computarizada como diagnóstico. Rev Cient Odontol. 2014; 2 (1): $224-28$
33. Proussaefs P, Lozada J, Kim J, Rohrer M. Repair of the perforated sinus membrane with a resorbable collagen membrane: a human study. Int J Oral Max Impl. 2004; 19 (3): 413-20.

34. Nolan PJ, Freeman K, Kraut R. Correlation between Schneiderian membrane perforation and sinus lift graft outcome: a retrospective evaluation of 359 augmented sinus. J. Oral Maxillofac. Surg. 2014; 72 (1): 47-52. doi: 10.1016/j.joms.2013.07.020

35. Kozuma A, Sasaki M, Seki K, Toyoshima T, Nakano H, Mori Y. Preoperative chronic sinusitis as significant cause of postoperative infection and implant loss after sinus augmentation from a lateral approach. J Oral Maxillofac Surg. 2017; 21 (2): 193-200. doi: 10.1007/s10006-017-0611-8

36. Moreno J, González A, Gil H, Mifsut R. Complication rate in 200 consecutive sinus lift procedures: guidelines for prevention and treatment. J Oral Maxillofac Surg. 2014; 72 (5): 892-901. doi: 10.1016/j.joms.2013.11.023

37. Khoury F, Javed F, Romanos G. Sinus augmentation failure and postoperative infections associated with prophylactic clindamycin therapy: an observational case series. Int J Oral Max Impl. 2018; 33 (5): 1136-9. doi: 10.11607/jomi.6517

38. Ayna M, Gülses A, Mede I. Management of infection after grafting of the sinus bone. Br J Oral Maxillofac Surg. 2017; 55 (2): 198-9. doi: 10.1016/j.bjoms.2016.06.021

39. Ding X, Wang Q, Guo X, Yu Y. Displacement of a dental implant into the maxillary sinus after internal sinus floor elevation: report of a case and review of literature. Int J Clin Exp Med. 2015; 8 (4): 4826-36.

40. Manor Y, Anavi Y, Gershonovitch R, Lorean A, Mijiritsky E. Complications and management of implants migrated into the maxillary sinus. Int J Periodont Rest. 2018; 38 (6): e112-e118. doi: $10.11607 /$ prd.3328

41. Galindo P, Sánchez E, Ávila G, Cutando A, Fernández J. Migration of implants into the maxillary sinus: two clinical cases. Int J Oral Max Impl. 2005; 20 (2): 291-5. 